\title{
Social Media Use and Prevention of HIV and Other Sexually Transmitted Infections among At-Risk College Students in the United States
}

\author{
Jacob J. van den Berg \\ Brown University School of Public \\ Health \\ Jacob_Vandenberg@Brown.edu
}

\author{
Don Operario \\ Brown University School of Public \\ Health \\ Don_Operario@Brown.edu
}

\author{
John C. Barnhart \\ The Miriam Hospital \\ JBarnhart@Lifespan.org
}

\author{
Benjamin Grin \\ Brown University School of Public \\ Health \\ Benjamin_Grin@Brown.edu
}

\author{
Beth C. Bock \\ Alpert Medical School Brown \\ University \\ Beth_Bock@Brown.edu
}

\begin{abstract}
The purpose of this study was to evaluate college students' self-perceptions of HIV/STI risk, potential barriers to HIV/STI testing, use of social media, and technology-based HIV/STI health interventions.

Surveys were administered to 97 US college students. Participants were categorized into three groups based on sexual behaviors: (1) men who have sex with men (MSM), (2) men who have sex with women (MSW), and (3) women who have sex with men (WSM).

MSM (n=24) were significantly more likely MSW/WSM $(n=72)$ to report being tested in the past year for HIV $(p<.01)$ and other STIs $(p<.01)$. Only $35 \%$ reported HIV testing and $24 \%$ reported STI testing in the past year. MSM were more likely than $M S W$ to report having met a sexual partner through social media $(p<.01)$, while no WSM reported doing so. The average number of partners met online in the past year was 7.8 (range=1-20). Those who had met a partner online were more willing to receive e-mail or text message HIV/STI testing reminders ( $p<.05)$.
\end{abstract}

\section{Introduction}

Sexually transmitted infections (STIs) encompass a diverse array of pathogens that collectively result in approximately 20 million new cases of infection annually in the United States (US), with half of those occurring in youth ages $15-25$, according to a report by the Centers for Disease Control and Prevention (CDC) [1]. This is particularly distressing as STIs cause significant morbidity and often go undetected [2]. Chlamydia trachomatis represents the most widespread infection, with a total of $1,412,791$ cases of genital
Chlamydia reported in 2011 [1]. Along with Neisseria gonorrhoeae, another common STI, Chlamydia presents a threat to females of childbearing age, with potential complication including pelvic inflammatory disease, infertility, and ectopic pregnancy [2]. Syphilis was once targeted for elimination in the US, after declining $89.7 \%$ from $1990-2000$, yet rates have increased more recently, particularly among young men who have sex with men (YMSM). Syphilis is concerning due to its potential to increase susceptibility to HIV infection and the possibility of fetal infection in untreated pregnant women [3]. In addition to human suffering, STIs place a significant burden on the US economy, contributing to nearly $\$ 16$ billion to annual health care costs [1]. The CDC recommends routine annual screening of all sexually active females under age 25 for Chlamydia and Gonorrhea, while routine syphilis screening of asymptomatic adolescents is recommended only for those considered high-risk, such as YMSM [4].

Of all the STIs, HIV, remains a significant problem in the US, with new cases disproportionately affecting YMSM of African American and Hispanic/Latino descent [5]. A 2011 CDC report found that young people aged 20-24 had the highest number and rate of HIV diagnoses of any group, with 36.9 new HIV diagnoses/100,000 people [5]. In Rhode Island, rates of syphilis, gonorrhea, and chlamydia have increased significantly in the last several years, especially among younger adults [6] and the rate of new HIV infections among college students have been well-documented [7]. Additional studies suggest that college students, particularly YMSM, may be at greater risk of HIV infection than previously thought [8, 9]. This parallels trends among MSM overall, who constitute most new HIV infections reported, both 
nationally and locally $[6,10]$. The CDC's revised recommendations call for routine opt-out HIV screening of all adolescents and adults in all health care settings [11].

Public health officials need avenues by which to reach at-risk groups with interventions aimed at combating HIV and other STIs. Newly developed empirically-based interventions targeted to college students are virtually non-existent despite widespread evidence that approximately $80 \%$ of college students are sexually active and many engage in risky sexual behaviors, including having multiple sexual partners and inconsistently using condoms [12-15]. In addition, almost $65 \%$ of undergraduates in one study reported using alcohol in the last 30 days and of those who drank over $20 \%$ reported having unprotected sex when drinking in the last 12 months [12]. In a different study, only $26.4 \%$ of sexually active college students reported always using condoms [14]. Given that one in two sexually active young people will contract an STI by age 25 , more effective interventions are needed [13].

Online social media services and mobile communication technologies are highly utilized by youth across all racial groups [16, 17]. As of 2010, $75 \%$ of young adults aged 18-29 reported using social networking sites, such as Facebook, MySpace, and Google Plus [16]. The same study found that text messaging is widespread among 18-29 year olds, with $88 \%$ reporting using text messaging and, of those, $80 \%$ texting a median of 20 times in the past 24 hours [16]. These information and communication modalities can also be effective avenues for public health interventions, referred more commonly as eHealth [18]. eHealth is an emerging and highly promising strategy for improving the reach of public health campaigns - and refers to a range of electronic technologies (e.g., Internet, telecommunications) for facilitating health communications to target audiences. mHealth is one specific eHealth approach based on the use of wireless technology, such as mobile telephone devices, for delivering public health and HIV/STI prevention information. Young adults often use the internet for health information, with $72 \%$ of those aged 18-29 reporting having done so in one study [19]. A randomized controlled trial of e-mail- and text message-based sexual health promotion messages among young people aged 16-29 in Australia found that STI knowledge improved significantly in the intervention group for both sexes compared to the control [20]. Females, but not males, in the intervention group were more likely to report getting an STI test and discussing their sexual health with a healthcare provider than those in the control group [20]. A recent review of eHealth interventions found a limited number of studies on computer and Internetbased interventions, including social networking site interventions [21-24]. The Youthnet trials sought to demonstrate the efficacy of website interventions for primary HIV prevention among 18-24 year olds, yet the authors found that the results did not support their hypothesis that a short-duration internet-based intervention could substantially impact condom use [21]. However, the single-session study lacked repetition of its message and the authors suggested that interventions may be more successful when integrated with websites that target groups already visit [21]. Other studies of computer- and internet-based interventions have found evidence that such interventions can significantly improve safer sex knowledge, attitudes, self-efficacy, and other theoretical mediators of safer sex, as well as behavioral measures such as condom use, with better targeting and tailoring capabilities [24]. Gold and colleagues found that social networking sites, primarily Facebook, are being utilized for sexual health promotion, but these efforts are understudied and underreported in the scientific literature [23].

Given the challenges of social stigma and need for discretion, many YMSM use the internet to find sexual partners with resultant risky sexual behavior [24, 25]. Young and Rice found that among homeless youth in Los Angeles, California, online social networking usage was associated with both increases and decreases in sexual risk behavior [26]. The same study found that gay, lesbian, and bisexual homeless youth were more likely than other youth to meet a sexual partner online [26]. A qualitative study exploring online sexual networking among gay and bisexual men found that focus group participants use both services directed at a general audience and gay-specific services [25]. There has been a proliferation of web- and smartphone appbased services geared at helping gay and bisexual men find willing sexual partners located in close proximity to them. For instance, Grindr is a GPS-enabled app for Android and iOS smartphone platforms that displays personal profiles and pictures of MSM within the immediate vicinity of the user. Grindr alone has over 750,000 users in 162 countries, with about 500,000 of those located in the US [25].

Previous research on meeting sexual partners online has shown increased concurrent high-risk behaviors and lower reported condom use with online partners across several demographics, especially among YMSM [27-30]. A feasibility and efficacy study of a web-based syphilis-screening program targeted to MSM detected a significantly higher percentage of men who required treatment than did the clinic-based STI screening program [31]. Online chat room interventions, specifically focused on reaching 
MSM, are another area of eHealth that has been explored. One study of a chat room-based intervention among MSM found that $15 \%$ of individuals reported having had an HIV test at post-test and those individuals who reported having sex with both men and women had nearly six times the odds of reported being tested for HIV after the intervention [32]. Unlike offline interventions, online interventions can reach a large audience with comparatively fewer resources once established [33]. The current literature shows that eHealth interventions can be effective, particularly when integrated with existing online social media habits. Thus, a more thorough understanding of the technology usage habits of today's college students, who may be at-risk for HIV and other STIs, will enable a better-targeted public health response. In the present study, we aimed to: (a) evaluate attitudes and behaviors related to HIV and other STIs among at-risk college students; (b) describe college students' perceived barriers to enacting risk-reduction behaviors and to seeking HIV/STI testing; (c) explore technology and online social media usage habits among a college student population, particularly as they relate to sexual behaviors; and (d) assess college students' willingness to use technology-based HIV/STI testing interventions

\section{Methods:}

\subsection{Methods Overview}

We performed a one-time, anonymous survey of college students in the northeastern US. Questions pertained to sexual behaviors, HIV/STI-related behaviors and attitudes, use of communication technologies, and attitudes towards hypothetical public health interventions. Given the descriptive nature of the study, no specific a priori hypotheses were generated. The purpose of this study was to characterize social media use among a population of college students and, in context with their reported sexual health attitudes and behaviors, to explore technology-based modalities for possible use in future HIV/STI interventions aimed at at-risk college students.

\subsection{Participants}

One-hundred survey participants were recruited in-person during events held at a private liberal arts college in the northeastern US between October 2012 and February 2013. Events were sponsored by campusbased student-run organizations who gave permission for research staff to approach students potentially interested in completing a survey. Inclusion criteria were: (a) 18 years of age or older; (b) currently enrolled or affiliated with an institution of higher education; and (c) ability to understand English. Participants gave informed consent using a written consent document that was unsigned to protect anonymity, per a procedure approved by The Miriam Hospital Institutional Review Board. Participants were instructed not to write their name or other identifying information on the survey and asked to return the completed survey in a sealed envelope to research staff. A \$5 gift card was given to participants as compensation for completing the survey.

\subsection{Surveys}

The survey included questions about demographics, sexual behaviors, condom use, HIV/STI attitudes and testing behaviors, as well as technology and social media usage. Demographic characteristics included age, gender, sex at birth, race, ethnicity, student status, current living situation, college year, state lived in for most of the year, relationship status, and sexual orientation. Sexual behavior questions included the number of sexual partners for oral, vaginal, and anal sex in the past year. Receptive anal and insertive anal sex was asked separately about participant's male partners. Participants were also asked to report the number of online partners met for each sexual activity.

Specific questions about condom use for each sexual activity, condom use barriers, asking sexual partner about HIV status, strategic positioning, where sexual partner was met (online and offline venues), substance use at time of sex, engagement in transactional sex or sex that is predicated on actual or anticipated material gain (e.g., money, shelter, material goods, transportation), and injection drug use were also asked. HIV/STI attitudes and testing behaviors included separate questions about ever being tested for HIV and STIs, receipt of HIV and STI diagnosis, and date of most recent HIV and STI test. Self-perceived HIV and STI risk was assessed using a 6-point Likerttype scale with higher scores $(6=$ "high risk" and $1=$ "no risk") indicative of greater self-risk perception. Questions about barriers and facilitators to HIV and other STI testing included asking participants to select out of a list of responses (e.g., cost, feeling uncomfortable asking his/her provider, forgetting to ask his/her provider, afraid of results, and availability) the major barrier preventing them from getting tested for HIV and other STIs, and to check all that apply from a list of facilitators that encourage testing. Participants could also select the "other" category to write in specific barriers and facilitators to HIV and other STI testing. 
Questions about pre- and post-exposure prophylaxis (PrEP and PEP) were also asked to determine if participants had ever heard of PrEP and PEP, from whom (e.g., a health care provider, friend or family member) they had heard about PrEP and PEP, if they had ever taken PrEP or PEP, whether or not they would consider taking PrEP or PEP in the future, and what their major concern regarding the use of PrEP or PEP might be for themselves.

Technology and social media usage included questions about the use and frequency of text messages, mobile phone internet access and number of hours of mobile phone use, smartphone and app use, computer internet access and number of hours of online computer use, social media services used and the number of hours on individual social media sites. One question asked about how likely participants would be willing to receive messages reminding them about getting testing for HIV or other STIs that were delivered in different ways, such as through e-mail, text messaging, and social networking sites. Participants were asked if they had met sexual partners online and if they had answered questions about the gender of the partner, where they had met them, and any other characteristics of the sexual partner they had met online, such as if they had known them before, whether they were a student, the sexual partner's general age, where the sexual partner was from and where they met to hook-up, if they asked the sexual partner's HIV status and if they had engaged in strategic positioning, alcohol use, or drug use. Participants were also asked to rank how likely they would be willing to receive HIV/STI testing reminders via a range of different modalities including e-mail, text message, websites, smartphone apps, social networking sites, microblogging services, phone call, and chat room/forum using 4-point Likert-type scale, from 1= "Not Likely" to 4= "Extremely Likely."

\section{Analyses}

All statistical analyses were conducted using IBM SPSS Statistics version 20.0. To facilitate comparison based on risk, participants were categorized into three groups based on reported sexual behaviors: (1) MSM; (2) men who have sex with women (MSW); and (3) women who have sex with men (WSM). These groups were chosen based on the greater relative risk for HIV and some STIs among MSM compared to exclusively heterosexual populations demonstrated in other studies [34, 35]. Categorization into MSM and MSW/WSM groups was made first based on reported sexual behavior in the past year, and secondly based on reported sexual orientation, when sexual history in the past year was absent. Male respondents with any same- sex behaviors were categorized as MSM, as were gay/homosexual or bisexual identified males. Participants who identified as "queer", "questioning", or "not sure" were categorized based on sexual behaviors in the past year alone. Groups were compared for differences in responses to other survey questions, such as sexual risk behaviors and acceptability of technology-based interventions. Fisher's exact test was used for categorical variables and t-tests were used for continuous variables. Levene's test for equality of variances was used on continuous data prior to applying the independent samples t-test. P-values less than .05 were considered significant.

\section{Results}

\subsection{Participant Characteristics}

Of the 100 individuals screened for the study, 97 met study eligibility criteria and completed the survey (Table 1). Twenty-five percent were MSM and 74\% were MSW or WSM. One female participant who identified as gay/homosexual and reported exclusively female partners in the past year was excluded from the analysis of both groups, but was included in the total number of participants. The mean age of the total sample was 19.6 years $(S D=1.4$, range $=18-24$ years), the mean age of the MSM group was 20.0 years $(S D=1.5$, range $=18-24$ years $)$ and the mean age of the MSW/WSM group was 19.4 years $(S D=1.3$, range $=18-23$ years $)$. Sex at birth for the total sample included $52 \%$ females and $49 \%$ males. All participants who were MSM identified as male, while the MSW/WSM group was $68 \%$ female and $32 \%$ male. Reported sexual orientation was predominately straight/heterosexual (70\%). One participant categorized as MSM reported a straight/heterosexual orientation. The majority of participants identified as White (78\%) followed by Asian (17\%), Mixed or Multiple Races (11\%), Black (7\%) and other $(7 \%)$. Eighteen percent of the total sample identified as Hispanic/Latino. All undergraduate classes were well represented in the sample.

\subsection{Attitudes and Behaviors Related to HIV/STIs}

Table 1 summarizes the risk behaviors reported by our study cohort. MSM were significantly more likely than MSW/WSM (46\% versus 3\%, respectively; $p<$ .001) to report having met a sexual partner online. All of those participants who reported meeting a partner online were male. The $11 \mathrm{MSM}$ who reported meeting partners online met an average of 8.1 partners online in the past year $(S D=8.7)$, while the same number for the two MSW was 6.5 partners $(S D=7.8)$. Twenty-three 
percent reported that their last sexual partner met online was greater than four years older than themselves, $9 \%$ met a partner two to four years older, while the remaining $64 \%$ reported meeting a partner about the same age or younger. Of the total sample, $58 \%$ reported using a condom during their most recent sexual encounter. This did not differ significantly between the groups, although people who reported meeting partners online tended to also report using a condom during their most recent encounter $(75 \%)$. Participants were least likely to report always using condoms during oral sex, with only $1 \%$ doing so. Forty-nine percent reported always using condoms during vaginal sex. For anal sex, $62 \%$ reported always using condoms; MSM comprised the majority (71\%) of this group. There were no significant differences between groups in the proportion reporting always using condoms.

Table 1. Risk Behavior by Sexual Behavior Group

\begin{tabular}{|c|c|c|}
\hline Behavior & $\begin{array}{l}\text { MSM } \\
(\mathrm{n}=24)\end{array}$ & $\begin{array}{c}\text { MSW/WSM } \\
(\mathrm{n}=68)\end{array}$ \\
\hline Met partner online & $46 \%$ & $3 \%$ \\
\hline $\begin{array}{l}\text { Condom use - most recent } \\
\text { encounter }\end{array}$ & $52 \%$ & $61 \%$ \\
\hline Condom use "always" & $\%(\mathrm{n})$ & $\%(\mathrm{n})$ \\
\hline Vaginal sex & $100 \%(3)$ & $47 \%(53)$ \\
\hline Anal sex & $67 \%(15)$ & $50 \%(6)$ \\
\hline Oral sex & $0 \%(22)$ & $2 \%(56)$ \\
\hline Ask HIV status "always" & $39 \%$ & $16 \%$ \\
\hline Risk avoidance "always" & $33 \%$ & $16 \%$ \\
\hline Injection drug use & $0 \%$ & $1 \%$ \\
\hline Drug use during sex & $22 \%$ & $34 \%$ \\
\hline \multicolumn{3}{|l|}{ Transactional sex } \\
\hline Gave & $9 \%$ & $3 \%$ \\
\hline Received & $17 \%$ & $3 \%$ \\
\hline Any STI diagnosis & $9 \%$ & $1 \%$ \\
\hline \multicolumn{3}{|c|}{$\begin{array}{l}\text { Note. } * * p<.01 * p<.05 ; \text { MSM = Men Who Have Sex With } \\
\text { Men; MSW = Men Who Have Sex With Women; WSM = } \\
\text { Women Who Have Sex with Men; WSW = Women Who } \\
\text { Have Sex With Women; STI = Sexually Transmitted } \\
\text { Infection }\end{array}$} \\
\hline
\end{tabular}

Only $22 \%$ of the total sample reported always asking sexual partners about their HIV status. MSM were significantly more likely than MSW/WSM to report doing so (39\% versus $16 \%$, respectively; $p<$ $.05)$. Twenty percent reported that they "always" avoid certain sexual positions or behaviors to reduce risk (risk avoidance). MSM were more likely than MSW/WSM to report doing so, but the difference was not statistically significant $(33 \%$ versus $16 \%$, respectively; $p>.05)$. However, those who reported meeting a partner online were significantly more likely than those who had not to report "always" using such risk avoidance measures (46\% versus $15 \%$, respectively; $p<.05)$. There was a trend in which participants were more likely to "always" ask about HIV status with their online partners (58\%) compared to other times $(42 \%)$. Only $1 \%$ of the total sample reported injection drug use; however, 32\% reported using any illicit drug use during sex. Marijuana was the most commonly reported drug used during sex followed by ecstasy. A greater proportion of the total sample reported receiving money or a place to stay in exchange for sex (7\%) than those who reported giving money or a place to stay in exchange for sex (4\%). MSM were significantly more likely than MSW/WSM to report receiving money or a place to stay in exchange for sex (17\% versus 3\%, respectively; $p<$ $.05)$, as were those who reported meeting partners online compared to those who had not (23\% versus $4 \%$, respectively; $p<.05$ ). Three percent of the sample reported ever being diagnosed with an STI, with a greater proportion being MSM (9\%) compared to MSW/WSM (1\%) and those reporting meeting a partner online $(15 \%)$.

\subsection{HIV/STI Testing Attitudes, Barriers and Acceptability of Biomedical Prevention Strategies}

Thirty-five percent of participants reported being tested for HIV within the last 12 months, while only $26 \%$ reported being tested for any other STIs within the same time frame. MSM were significantly more likely than MSW/WSM to report having been tested for HIV ( $58 \%$ versus $28 \%$, respectively; $p<.05$ ) in the last 12 months. There was a trend in which MSM also reported being tested for other STIs in greater proportion than MSW/WSM but it was not statistically significant (39\% versus $21 \%$, respectively; $p>.05$ ).

All participants were asked to report perceived barriers to HIV/STI testing including cost, feeling uncomfortable asking his/her provider, forgetting to ask his/her provider, afraid of results, and availability. The majority of both groups reported having no barriers to HIV/STI testing (MSM $=68 \%$; MSW/WSM $=68 \%$ ). Of those who reported barriers, cost was the most frequently identified barrier for MSM (18\%), while MSW/WSM identified availability of testing/being unsure where to get tested as their top rated barrier (15\%). Most participants self-assessed risk for HIV as being "none" or "low," with the average scaled ranking for the total sample being 1.68 $(S D=0.74)$. There were no significant differences between the average scaled ranking of MSM and MSW/WSM. The average scaled ranking for perceived risk of other STIs was slightly greater in the total sample $(M=1.79 ; S D=0.8)$. For this item, MSM selfassessed a significantly greater average scale ranking 
than did MSW/WSM $(M=1.83 ; S D=0.65$ versus $M=$ 1.63; $S D=0.8$, respectively; $p<.01)$. The majority of participants reported discussing their sexual history with a medical provider $(68 \%)$, with no notable differences between MSM and MSW/WSM groups.

Few participants in either group were aware of the existence of post-exposure prophylaxis (PEP) or preexposure prophylaxis (PrEP) (18\% of MSM and $16 \%$ of MSW/WSM); however, $78 \%$ reported that they would consider taking PEP, while 56\% reported that they would consider taking PrEP. MSM were significantly more likely than MSW/WSM to report that they would consider taking PrEP (83\% versus $49 \%$, respectively; $p<.05)$. While the difference was not significant, MSM also reported that they would consider taking PEP in greater proportion than MSW/WSM (94\% versus $73 \%$, respectively; $p>.05$ ). Participants were asked why they did not always ask partners about their HIV status. The most commonly cited reason for non-disclosure was "They are people I know well" (41\%), followed by "I think it is awkward to ask" (28\%) and "I avoid certain high-risk sexual behaviors" (16\%). Individual write-in responses also suggested subjective judgments, such as socioeconomic status and assumed virginity, as reasons for non-disclosure.

\subsection{Technology and Online Social Media Use}

The use of text messaging was nearly ubiquitous, with $98 \%$ of the total sample reporting at least daily use. On average, participants reported sending/receiving 59 texts per day $(S D=90.7)$. Nearly all reported having an unlimited text-messaging plan (97\%). Most also reported owning a smartphone (84\%) and among those who did, owned an average of 22 smartphone apps $(S D=19.4)$. A similar proportion had internet access on their cell phone (83\%) and among these, the average daily use was 1.59 hours $(S D=2.0)$. Ninety-eight percent of participants reported owning their own computer with the remainder using public computers through which they could access the Internet. Participants reported using the Internet on a computer an average of 5.62 hours per day $(S D=3.1)$. Ninety percent reported checking e-mail "multiple times per day."

Participants' social media use was separated out by type. Ninety-three percent of all participants reported having an account on social networking sites (SNS). Eighty-seven percent reported visiting SNS "several times per day" and Facebook was the most frequent SNS where respondents had an account (95\%) followed by Google+ $(42 \%)$ and MySpace $(6 \%)$. Facebook was also the most engaging service by far with $79 \%$ of all participants reporting spending at least an hour on the site on days when they used it, while $17 \%$ spent four hours or more on the site. No other SNS had greater than $5 \%$ of participants who used the service one hour or more. Media sharing services, sites specializing in video or image sharing, were used by $76 \%$ of all participants with YouTube being most popular (73\%) followed by Pinterest (14\%). Blogging or microblogging services, social media sites primarily geared towards sharing long-form or short text content, were utilized by $55 \%$ of participants, including Twitter (45\%) and Tumblr (33\%). MSM were significantly more likely than MSW/WSM to have a blogging/ microblogging account (64\% versus 38\%, respectively, $p<05)$. Online dating services were analyzed separately based on whether they specialized in maleto-male dating. General dating services, excluding those targeting MSM, were used by $7 \%$ of the total sample with OKCupid being the most commonly cited (5\%). MSM used these services proportionality more than MSW/WSM (17\% versus 4\%, respectively; $p<$ 0.05). The smartphone app Grindr was most widely used male-to-male dating service $(7 \%)$ followed by Adam4Adam (4\%). Among the total sample, 5\% reported "Sexting" (sending/ receiving sexually explicit pictures) at least weekly, $10 \%$ used hook-up apps (like Grindr) at least weekly, and 5\% looked for sexual partners online at least weekly (Table 2).

Table 2: Social Media Use Percentage by Sexual Behavior Group and Online Partner History

\begin{tabular}{|c|c|c|c|}
\hline Social Media Type & $\begin{array}{l}\text { MSM \% } \\
(n=23)\end{array}$ & $\begin{array}{l}\text { MSW/WSM } \\
\%(n=72)\end{array}$ & $\begin{array}{l}\% \text { Met } \\
\text { online } \\
(\mathrm{n}=12)\end{array}$ \\
\hline Text message $>$ daily & 96 & 99 & \\
\hline Mean daily texts* & $54(55)$ & $61(100)$ & $58(95)$ \\
\hline Own smartphone & 74 & 87 & 67 \\
\hline Mean \# of apps * & $23(16)$ & $21(20)$ & $22(71)$ \\
\hline Mobile data plan & $74 \%$ & $86 \%$ & $58 \%$ \\
\hline $\begin{array}{l}\text { Mean daily use in } \\
\text { hours * }\end{array}$ & $1.78(2.3)$ & $1.53(1.8)$ & $1.52(1.7)$ \\
\hline $\begin{array}{l}\text { Internet Computer } \\
\text { mean daily use in } \\
\text { hours* }\end{array}$ & $\begin{array}{l}6.36 \\
(3.3)\end{array}$ & $\begin{array}{l}5.44 \\
(3.0)\end{array}$ & $\begin{array}{l}5.38 \\
(3.0)\end{array}$ \\
\hline \multicolumn{4}{|l|}{ Internet services } \\
\hline Social network & $96 \%$ & $94 \%$ & $83 \%$ \\
\hline Media sharing & $82 \%$ & $68 \%$ & $83 \%$ \\
\hline Blogging & $64 \%$ & $38 \%$ & $83 \%$ \\
\hline General dating & $17 \%$ & $4 \%$ & $33 \%$ \\
\hline Dating/Hook-up sites & $35 \%$ & $4 \%$ & $67 \%$ \\
\hline
\end{tabular}

*mean and (standard deviation)

\subsection{Willingness to Use HIV/STI eHealth Interventions}

Across all participants, the greatest proportion $(69 \%)$ were willing to receive reminders via e-mail followed by text message $(51 \%)$, websites $(38 \%)$, 
smartphone apps (35\%), social networking sites (34\%), microblogging services $(21 \%)$, phone call $(20 \%)$, and chat room/online forum $(13 \%)$. MSM reported greater willingness to receive HIV/STI testing reminders than the MSW/WSM group for most modalities (Table 4). This difference was statistically significant only for microblogging services (e.g., Twitter, Tumblr), where $40 \%$ of MSM reported they would be at least somewhat likely to accept testing reminders compared with $15 \%$ of MSW/WSM.

There were no statistically significant differences between females and males in willingness to receive HIV/STI testing reminders across all modalities tested. Males were slightly more willing to receive messages via social networking sites $(41 \%)$ than via smartphone app $(34 \%)$. Participants who had met a sexual partner online reported greater willingness to receive HIV/STI testing reminders via all modalities tested. When compared with those who never met a partner online, these differences were statistically significant $(p<.05)$ for all modalities except e-mail. Text messaging was the most acceptable modality among those who had met a partner online $(91 \%)$ followed by social networking sites (80\%) and e-mail (73\%). Finally, participants were asked how frequently they would be willing to receive HIV/STI testing reminders via a range of modalities. Participants who indicated that they were "not likely" to be willing to accept an intervention via a given modality were counted as desiring a frequency of "never" for that modality, regardless of the frequency they indicated. Participants were willing to receive reminders with the greatest frequency monthly or more often via e-mail (27\%), text messaging (24\%), smartphone app (20\%), and social networking sites $(20 \%)$.

\section{Discussion}

The present study characterized attitudes and behaviors of college students pertaining to HIV/STIs and use of social media as a potential intervention. Consistent with previous reports, our cohort of college students engaged in risky sexual behaviors [12, 14]. Despite this, low rates of HIV/STI testing were reported by participants with only $35 \%$ being tested for HIV and $26 \%$ being tested for other STIs in the last 12 months. This highlights the need for newly developed interventions to promote testing and prevention among college students who are sexually active. Social and other eHealth media use is high among college students. Most college students, including those at highest-risk, are willing to receive eHealth interventions to promote HIV testing and other interventions targeting HIV prevention.
Our cohort of college students was at-risk of HIV/STI infections due to having multiple sexual partners and inconsistent condom use. Some of our participants also reported engaging in transactional sex and drug use during sex. Previous research has found that being under the influence of illicit drugs or alcohol during sex is associated with unprotected receptive anal intercourse among YMSM [36]. Having a previous STI diagnosis was more common among the MSM group, although this finding may be confounded by the higher rates of testing reported by this group. Nearly half of MSM in our sample reported meeting a sexual partner online, with many of those reporting multiple such partners in the past year and older partners. Garofalo et al. [27] found that YMSM who met sexual partners online often engaged in behaviors that placed them at risk for HIV and other STIs. A survey of adult MSM by McFarlene and colleagues [29] also found that seeking sex partners online was associated with a previous STI diagnosis, having a greater number of sexual partners, and having sexual exposure to a person known to be HIV-positive. These concomitant risk factors, while not definitely linked to online sex seeking, suggest that HIV/STI prevention strategies should be developed for online sex seekers.

The college students in our sample reported low rates of testing for HIV and other STIs. This is consistent with previous research on New England college health center medical directors, who reported low rates of student HIV testing across the region [37]. The majority of students in our sample reported having no barriers to being tested, suggesting that they may simply lack motivation to do so. This finding is supported by the very low perceived risk of HIV and similarly low levels of self-assessed risk of other STIs among study participants. Adolescents and young adults as a group often exhibit an optimistic bias and perceive themselves to be invulnerable or invincible [38-40]. Pollack et al. [40] showed that perceived risk for STIs among young adult women does align with risk behavior, with the exception of condom non-use, but it is not clear what effect this may have on testing behavior. A different study among YMSM found that HIV/AIDS complacency, associated with knowledge of highly active antiretroviral therapy efficacy, predicted sexual risk behavior [41]. MSM in the present study self-assessed a higher risk of STIs besides HIV compared to MSW/WSM, and were more likely to have accessed HIV and other STI testing in the past year. This finding may reflect the effect of the targeted outreach to this community or a general awareness of elevated risk for gay and bisexual men among this educated population. However, both MSM and MSW/WSM groups were more likely to have accessed HIV testing in the past year than testing for 
other STIs. This is contrary to the relative incidence of HIV compared to other STIs as estimated by the CDC nationally [1] and the Rhode Island Department of Health locally [6]. The trend is concerning, in particular, for young women who could face significant sequelae from untreated Chlamydia and Gonorrhea infections [2]. However, it is possible that women in our sample failed to report screening conducted during routine gynecological visits, given that the majority reported discussing their sexual history with a medical provider.

Use of electronic communication technologies and online social media was very high among the college students surveyed. Text messaging, in particular, was nearly ubiquitous and heavily utilized by participants. Smartphones, mobile apps, and mobile internet connectivity were also common among this sample. Recent research has shown a narrowing of the "digital divide" between white and minority youth and the present study supports this trend [30]. However, our findings may also serve as an indicator of the higher socioeconomic status of our sample than in the general population.

Social media services, particularly Facebook and media sharing sites, were widely adopted by participants. The amount of time students reported using Facebook was striking, with $79 \%$ reporting spending at least an hour or more on the site every day. It is interesting that the MSM participants reported frequently (at least weekly) using hook-up apps like Grindr at twice the rate they reported frequently looking for sexual partners online. This is consistent with focus group data collected by Gudelunas on gay men exploring the different use cases of apps like Grindr as compared with general social networking sites (SNS) like Facebook [25]. In this study, Gudelunas found that gay-specific social network sites were used for multiple purposes, including facilitating sexual encounters and friendships between gay men. This dual purpose identified by Gudelunas may explain the gap observed in the present study between the use of such apps and intent to find a sexual partner. Gudelunas's study also offers insights into why MSM might utilize online dating services more often than MSW/WSM, given the cultural restriction and/or stigmatization of non-normative sex practices he identifies. In this light, electronic communication modalities may be viewed as an outlet for some people. In the present study, participants who met sexual partners online were more likely to use general dating services and male-to-male specific services. However, the partial sample size of those reporting meeting a partner online $(n=12)$ was deemed too small to support further analysis on behavioral risk factors with online partners.
Our findings also suggest that college students are willing to receive HIV/STI testing reminders via e-mail or text message. Notably, both are private, direct communication modalities that our participants report using extensively. A randomized controlled trial on the effect of a sexual health promotion program delivered via e-mail and text message among young adults in Australia found evidence of efficacy for such interventions [20]. A qualitative study among young black MSM found that smartphones would be an acceptable modality for HIV interventions [42]. MSM in our sample reported using microblogging services at a significantly greater rate than do non-MSM, which may explain why MSM reported being more willing to receive testing reminders using that modality.

It is also important to highlight that participants in the present study who had met a sexual partner online were significantly more willing to receive testing reminders via nearly all modalities investigated. While this may be an artifact due to the small size of the sample, we believe that it may be indicative of a genuinely greater acceptability of using social media for sexual health promotion among these young persons. Our work suggests that college students, in particular, may benefit from eHealth interventions because they are open to and comfortable with current technology. Thus, targeted public health interventions that utilize social media specifically developed for college students are warranted.

The strengths of the present study are that we evaluated current attitudes and behaviors of college students around HIV and other STIs, which are important given increasing rates of infection among young people. In addition, we describe college students' perceived barriers to enacting risk-reduction strategies and to seeking HIV/STI testing as a way of understanding how we may intervene to improve the health and well-being of young people. We also explore technology and online social media usage habits of a college student population to determine their acceptability of technology-based HIV/STI testing interventions.

Despite its strengths, there are potential limitations that must be considered before drawing inferences from the present study. First, participants were approached at campus-based, student-run events at a single institution, which may limit generalizability and bias the sample in favor of participants more willing to participant in research. Second, the partial sample sizes for MSM $(n=24)$ and those who met a sexual partner online $(n=12)$ were small and comparisons involving these groups should not be considered definitive. Compared to the most recent demographics statistics of the private liberal arts college in which participants were recruiting from for the present study, white and 
mixed-race students were overrepresented by $7 \%$ and $6 \%$, respectively. Third, our survey relied on participant self-report, which must be interpreted with caution.

\section{References}

[1] Centers for Disease Control and Prevention (CDC): Sexually transmitted disease surveillance 2011. [http://www.cdc.gov/std/stats11/default.htm]

[2] O'Connor CA, Shubkin CD: Adolescent STIs for primary care providers. Curr Opin Pediatr 2012, 24:647-55.

[3] CDC: Recommendations for public health surveillance of syphilis in the United States.

[http://www.cdc.gov/std/SyphSurvReco.pdf]

[4] CDC: Special populations - 2010 STD treatment guidelines.

[http://www.cdc.gov/std/treatment/2010/specialpops.htm]

[5] CDC: HIV among youth.

[http://www.cdc.gov/hiv/youth/pdf/youth.pdf]

[6] Rhode Island Department of Health. 2012 Rhode Island Epidemiologic Profile of HIV/AIDS.

[http://www.health.ri.gov/publications/epidemiologicalprofil es/2012HIVAIDSViralHepatitisWithSurrogateData.pdf]

[7] Chan PA, Kazi S, Rana, A, Blazar I, DeJong CC, Mayer KH, Huard TK, Carleton K, Gillani F, Alexander N, Parillo Z, Flanigan TP, Kantor R: New HIV infections in Southern New England college students: an emerging epidemic? AIDS Res Hum Retroviruses 2013, 29:25-9.

[8] Hightow LB, Leone PA, Macdonald PD, McCoy SI, Sampson LA, Kaplan AH: Men who have sex with men and women: a unique risk group for HIV transmission on North Carolina College campuses. Sex Transm Dis 2006, 33:58593.

[9] Hightow LB, MacDonald PD, Pilcher CD, Kaplan AH, Foust E, Nguyen TQ, Leone PA: The unexpected movement of the HIV epidemic in the Southeastern United States: transmission among college students. J Acquir Immune Defic Syndr 2005, 38:531-7.

[10] Hall HI, Song R, Rhodes P, Prejean J, An Q, Lee LM, Karon J, Brookmeyer R, Kaplan EH, McKenna MT, Janssen RS; HIV Incidence Surveillance Group: Estimation of HIV incidence in the United States. JAMA. 2008, 300:520-9.

[11] Branson BM, Handsfield HH, Lampe MA, Janssen RS, Taylor AW, Lyss SB, Clark JE; CDC: Revised recommendations for HIV testing of adults, adolescents, and pregnant women in health-care settings. MMWR Recomm Rep. 2006, 55(RR-14):1-17.

[12] American College Health Association (ACHA): ACHANational college health assessment II: Reference group data report spring 2012. [http://www.acha-ncha.org/docs/ACHANCHA-II_ReferenceGroup_DataReport_Spring2012.pdf]
[13] Boudewyns V, Paquin RS: Intentions and beliefs about getting tested for STDs: implications for communication interventions. Health Commun. 2011, 26:701-11.

[14] Certain HE, Harahan BJ, Saewyc EM, Fleming MF: Condom use in heavy drinking college students: the importance of always using condoms. J Am Coll Health. 2009, 58:187-94.

[15] Oswalt SB, Wyatt TJ: Sexual health behaviors and sexual orientation in a U.S. national sample of college students. Arch Sex Behav. 2013, 48:1561-72.

[16] Chiasson MA, Hirshfield S, Rietmeijer C: HIV prevention and care in the digital age. J Acquir Immune Defic Syndr. 2010, 55 Suppl 2:S94-7.

[17] Lindstrom Johnson S, Tandon SD, Trent M, Jones V, Cheng TL: Use of technology with health care providers: perspectives from urban youth. J Pediatr. 2012, 160:9971002.

[18] Eng TR: The eHealth landscape: a terrain map of emerging information and communication technologies in health and health care.

[http://www.hetinitiative.org/media/pdf/eHealth.pdf]

[19] Lenhart A, Purcell K, Smith A, Zickuhr K: Social media $\&$ mobile internet use among teens and young adults. [http://files.eric.ed.gov/fulltext/ED525056.pdf]

[20] Lim MS, Hocking JS, Aitken CK, Fairley CK, Jordan L, Lewis JA, Hellard ME: Impact of text and email messaging on the sexual health of young people: a randomised controlled trial. J Epidemiol Community Health. 2012, 66:6974.

[21] Bull S, Pratte K, Whitesell N, Rietmeijer C, McFarlane M: Effects of an Internet-based intervention for HIV prevention: the Youthnet trials. AIDS Behav. 2009, 13:47487.

[22] Gold J, Lim MS, Hocking JS, Keogh LA, Spelman T, Hellard ME: Determining the impact of text messaging for sexual health promotion to young people. Sex Transm Dis. 2011, 38:247-52.

[23] Gold J, Pedrana AE, Sacks-Davis R, Hellard ME, Chang S, Howard S, Keogh L, Hocking JS, Stoove MA: A systematic examination of the use of online social networking sites for sexual health promotion. BMC Public Health. 2011, 11:583.

[24] Noar SM, Willoughby JF: eHealth interventions for HIV prevention. AIDS Care. 2012, 24:945-52.

[25] Gudelunas D: There's an app for that: the uses and gratifications of online social networks for gay men. Sex Cult. 2012, 16:347-65.

[26] Young SD, Rice E: Online social networking technologies, HIV knowledge, and sexual risk and testing behaviors among homeless youth. AIDS Behav. 2011,15:25360. 
[27] Garofalo R, Herrick A, Mustanski BS, Donenberg GR. Tip of the Iceberg: young men who have sex with men, the Internet, and HIV risk. Am J Public Health. 2007, 97:1113-7.

[28] Kalichman SC, Cherry C, Cain D, Pope H, Kalichman M. Psychosocial and behavioral correlates of seeking sex partners on the internet among HIV-positive men. Ann Behav Med. 2005, 30:243-50.

[29] McFarlane M, Bull SS, Rietmeijer CA. The Internet as a newly emerging risk environment for sexually transmitted diseases. JAMA. 2000, 284:443-6.

[30] Whiteley LB, Brown LK, Swenson RR, Valois RF, Vanable PA, Carey MP, DiClemente R, Salazar LF, Romer D. African American adolescents meeting sex partners online: closing the digital research divide in STI/HIV prevention. J Prim Prev. 2012, 33:13-8.

[31] Koekenbier RH, Davidovich U, van Leent EJ, Thiesbrummel HF, Fennema HS: Online-mediated syphilis testing: feasibility, efficacy, and usage. Sex Transm Dis. 2008, 35:764-9.

[32] Rhodes SD, Vissman AT, Stowers J, Miller C, McCoy TP, Hergenrather KC, Wilkin AM, Reece M, Bachmann LH, Ore A, Ross MW, Hendrix E, Eng E: A CBPR partnership increases HIV testing among men who have sex with men (MSM): outcome findings from a pilot test of the CyBER/testing internet intervention. Health Educ Behav. 2011, 38:311-20.

[33] Rietmeijer CA, McFarlane M: STI prevention services online: moving beyond the proof of concept. Sex Transm Dis. 2008, 35:770-1.

[34] Finlayson TJ, Le B, Smith A, Bowles K, Cribbin M, Miles I, Oster AM, Martin T, Edwards A, Dinenno E; CDC: HIV risk, prevention, and testing behaviors among men who have sex with men--National HIV Behavioral Surveillance System, 21 U.S. cities, United States, 2008. MMWR Surveill Summ. 2011, 60:1-34.

[35] Pathela P, Braunstein SL, Schillinger JA, Shepard C, Sweeney M, Blank S: Men who have sex with men have a 140-fold higher risk for newly diagnosed HIV and syphilis compared with heterosexual men in New York City. J Acquir Immune Defic Syndr. 2011, 58:408-16.

[36] Celentano DD, Valleroy LA, Sifakis F, MacKellar DA, Hylton J, Thiede H, McFarland W, Shehan DA, Stoyanoff SR, LaLota M, Koblin BA, Katz MH, Torian LV; Young Men's Survey Study Group: Associations between substance use and sexual risk among very young men who have sex with men. Sex Transm Dis. 2006, 33:265-71.

[37] Patel N, Rana A, Thomas A, Barnhart JC, Flanigan TP, van den Berg JJ, Chan PA: HIV testing practices among New England college health centers. AIDS Res Ther. 2013, 10:8.

[38] Millstein SG, Halpern-Felsher BL: Judgments about risk and perceived invulnerability in adolescents and young adults. J Res Adolesc. 2002, 12:399-422

[39] Weinstein ND: Unrealistic optimism about susceptibility to health problems: conclusions from a community-wide sample. J Behav Med. 1987, 10:481-500.

[40] Pollack LM, Boyer CB, Weinstein ND: Perceived risk for sexually transmitted infections aligns with sexual risk behavior with the exception of condom nonuse: data from a nonclinical sample of sexually active young adult women. Sex Transm Dis. 2013, 40:388-94.

[41] Mackellar DA, Hou SI, Whalen CC, Samuelsen K, Valleroy LA, Secura GM, Behel S, Bingham T, Celentano DD, Koblin BA, LaLota M, Shehan D, Thiede H, Torian LV: A plausible causal model of HAART-efficacy beliefs, HIV/AIDS complacency, and HIV-acquisition risk behavior among young men who have sex with men. AIDS Behav. 2011, 15:788-804.

[42] Muessig KE, Pike EC, Fowler B, LeGrand S, Parsons JT, Bull SS, Wilson PA, Wohl DA, Hightow-Weidman LB: Putting prevention in their pockets: developing mobile phone-based HIV interventions for black men who have sex with men. AIDS Patient Care STDS. 2013, 27:211-22. 